\title{
Brain abscess in a non-penetrating traumatic intracerebral hematoma: Case report and review of literature
}

\author{
Santhosh George Thomas, Ranjith K. M oorthy, V edantam Rajshekhar \\ Department of Neurological Sciences, Christian Medical College, Vellore, India
}

\author{
Address for correspondence: \\ Dr. Vedantam Rajshekhar, \\ Department of Neurological \\ Sciences, Christian Medical College, \\ Vellore, Tamil Nadu, India. \\ E-mail: rajshekhar@ cmcvellore.ac.in
}

DOI: $10.4103 / 0028-3886.48812$

\begin{abstract}
Abstrat
We report a 57 -year-old man who presented one month after sustaining a traumatic right temporal intracerebral hematoma with history of headache, left hemiparesis and altered sensorium of two days duration. A diagnosis of right temporal resolving hematoma was made on computed tomography scan. However, his sensorium progressively deteriorated and he underwent craniotomy and partial excision of an abscess. He was treated with appropriate antibiotics for six weeks despite of which he did not improve and died nine months later. We conclude that there should be a high index of suspicion for brain abscess in patients with traumatic intracerebral hemorrhage if the clinical and radiological picture is different from the expected course of a resolving hematoma.
\end{abstract}

Key words: Brain abscess, intracerebral hemorrhage, trauma

\section{Introduction}

Brain abscess is a neurosurgical emergency that necessitates urgent surgical evacuation and treatment with appropriate antibiotics. Brain abscess is mostly due to hematogenous spread from a distant focus of infection. ${ }^{[1]}$ Absence of a clear source of infection is reported in as many as $40 \%$ of cases. ${ }^{[2]}$ Brain abscess complicating intracerebral hemorrhage is rare and to our knowledge, only 18 cases have been reported so far and none was post- traumatic in origin. Almost all these patients had episodes of bacteremia, sepsis, or local infection such as phlebitis or an infected surgical wound. ${ }^{[3-9]}$ Our patient developed the abscess in a traumatic intracerebral hematoma with no identifiable focus of infection.

\section{Case Report}

A 57-year-old man presented with history of headache, left hemiparesis and altered sensorium of two days duration. He sustained a fall one month prior to this admission following which he developed a right temporal intracerebral hematoma. He was managed conservatively with antiepileptics and antiedema measures. He was a diabetic on irregular medication. On examination, the glasgow coma scale (GCS) score was 13/15 and pupils were equal and reacting. There was Grade $4 / 5$ power in the left upper and lower limbs. There was no evidence of any external injury.

Plain computed tomography (CT) scan of the brain done three days following the fall showed a right temporal hematoma with mild mass effect and no midline shift [Figure 1] Contrast CT done at the time of this admission showed an intra-axial lesion in the same location with mild perilesional edema which was thought to be a resolving hematoma with an enhancing rim [Figure 2]. There was a mild increase in the mass effect but it did not warrant surgical evacuation. CT angiogram was negative for an aneurysm. He was managed conservatively with antiepileptics and antiedema measures. On the second hospital day, the GCS score started fluctuating between 10/15 (E3V2M5) to 13/15 (E3V4M6). There were no seizures, fever, meningismus or focus of any infection. The total leucocyte count was $8300 / \mathrm{mm}^{3}$. A repeat plain CT scan brain [Figure 3] showed increase in the perilesional edema and mass effect. He underwent right temporal craniotomy and partial excision of lesion on the fifth 


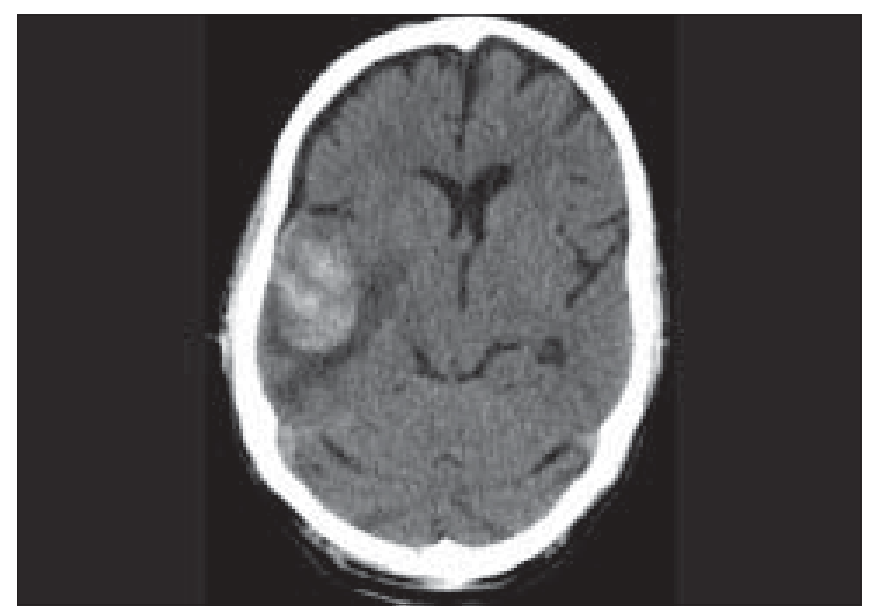

Figure 1: Axial section of a non-contrast CT of the brain three days following trauma showing a right temporal hematoma with mild mass effect and no midline shift

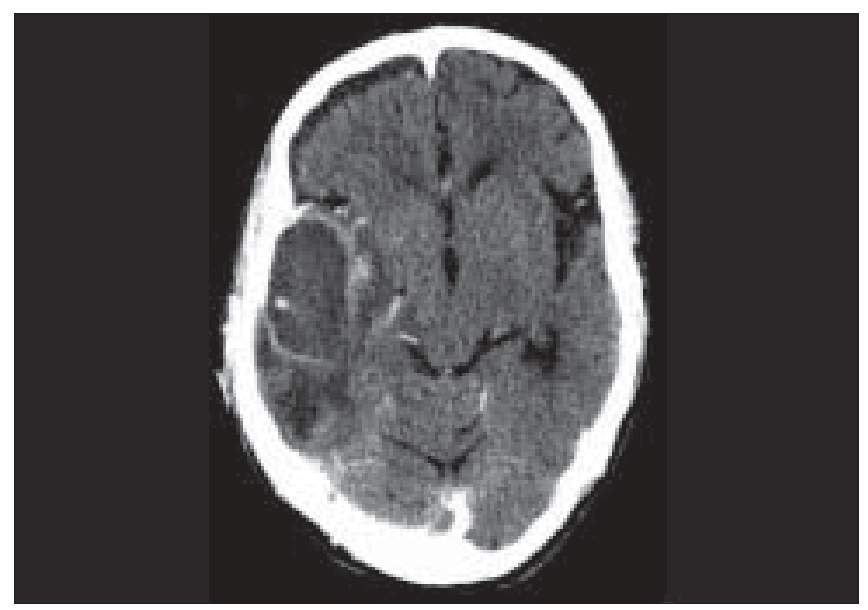

Figure 2: Axial section of a contrast CT of brain one month after trauma showing an intra-axial lesion in the right temporal lobe with mild perilesional edema thought to be a resolving hematoma with an enhancing rim

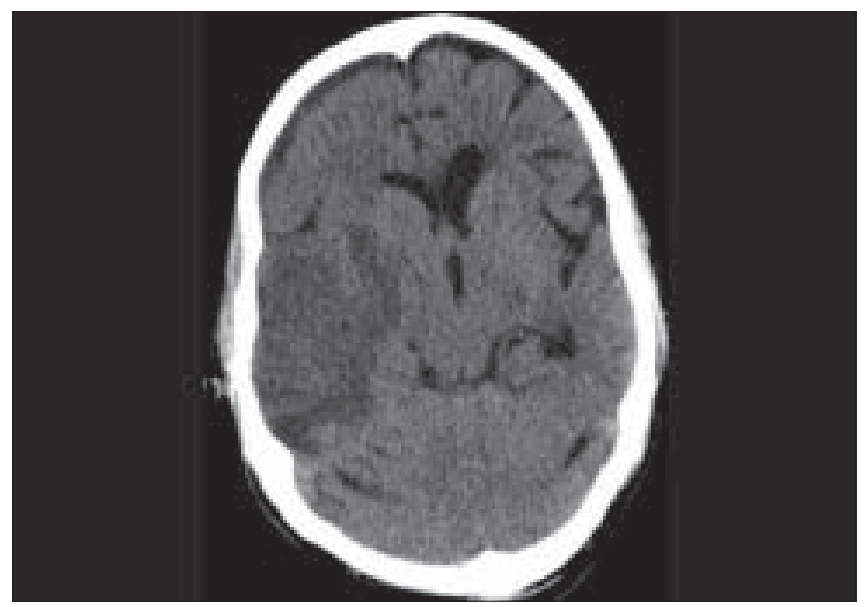

Figure 3: Axial section of a non-contrast CT of the brain five days after admission showing an increase in the perilesional edema and mass effect hospital day. There was a well-encapsulated collection of pus mixed with blood in the superior temporal gyrus with secondary adhesions to the overlying dura. The temporal horn of the lateral ventricle was opened into during the excision. The pus grew Escherichia coli and he was treated with Cefotaxime and Amikacin for six weeks based on sensitivity. Echocardiogram, chest radiographs, otolaryngological and dental examinations were negative for a potential source of infection. Postoperative CT scan showed pneumocephalus with minimal blood within the lateral ventricle. However, the GCS persistently remained at 9/15 (E2V2M5) with resolution of the left hemiparesis. The persistent low GCS was thought to be due to ventriculitis as the temporal horn was entered into during surgery. He was discharged on the tenth postoperative day for further management to a secondary level hospital. His GCS score remained the same and he died after nine months.

\section{Discussion}

\section{Epidemiology and microbiological spectrum}

The incidence of brain abscesses is $\sim 8 \%$ of intracranial masses in developing countries, whereas in the West the incidence is $\sim 1-2 \%$. Mortality rates have reduced with the advent of CT and effective antibiotics. ${ }^{[1,3]}$ About $30 \%$ of the patients who survive can be left with neurological deficits and symptomatic seizures. ${ }^{[4]}$ The common predisposing factors for brain abscess include synchronous infection in the middle ear or paranasal sinuses, cyanotic heart disease, diabetes mellitus and immunosuppression. ${ }^{[1,3]}$ Several organisms (mostly gram-positive cocci) have been reported as pathogens in an abscess at the site of a recent spontaneous intracerebral hematoma and the details have been summarized in Table 1.

\section{Pathogenesis and plausible mechanisms}

An intracerebral hematoma disrupts the blood-brain barrier, raising the susceptibility for inoculation of bacteria through the hematogenous route. ${ }^{[3]}$ Vasculitis is also reported to be a cause of granulomatous inflammation and fibrinoid necrosis of vessel walls that causes disruption of leptomeningeal and parenchymal arteries and veins leading to hemorrhages and disruption of the blood-brain barrier. ${ }^{[2]}$ Rupture of mycotic aneurysms in patients with infective endocarditis may lead to hematoma which can become secondarily infected. ${ }^{\left[{ }^{[6]}\right.}$ Most reported cases of abscesses at the site of a recent spontaneous intraparenchymal hematoma had episodes of high fever indicating bacteremia that occurred approximately two to three weeks after the bleed. ${ }^{[3-7]}$

Our patient was a diabetic on irregular medication and did not have any other risk factors and there was 


\begin{tabular}{|c|c|c|c|}
\hline Author and year & Site & Focus of infection & Pathogen \\
\hline Israel et al. ${ }^{[3]}$ & Left basal ganglia & Unknown & Pneumococcus \\
\hline Biller et al. ${ }^{[8]}$ & Right frontal lobe & Wound infection & Staphylococcus sp. \\
\hline Biller et al. ${ }^{[8]}$ & Right basal ganglia & Episiotomy & Staphylococcus sp. \\
\hline Kurihara et al. ${ }^{[5]}$ & Right putamen & Phlebitis & Staphylococcus sp. \\
\hline Mashimoto et al. ${ }^{[3]}$ & Right basal ganglia & Endocarditis & Enterococcus Fecalis \\
\hline Lee et al. ${ }^{[5]}$ & Right putamen & Sepsis & Streptococcus sp. \\
\hline Lida and Takemura ${ }^{[5]}$ & Cerebellum & Pneumonia & Staphylococcus sp. \\
\hline Chen et al. ${ }^{[7]}$ & Left putamen & Pneumonia & Klebsiella \\
\hline Bert et al. ${ }^{[3]}$ & Left temporal & Phlebitis & Staphylococcus sp. \\
\hline Sumoika et al. ${ }^{[3]}$ & Right putamen & Dental infection & Morgananella morgani \\
\hline Okami et al. ${ }^{[5]}$ & Left thalamus & Sepsis & Staphylococcus sp. \\
\hline Inamasu et al. ${ }^{\left[{ }^{9]}\right.}$ & Right putamen & Pneumonia & Enterococcus faecalis \\
\hline Amayo et al. ${ }^{[6]}$ & Left basal ganglia & Pneumonia & Sterile \\
\hline Nowak et al. ${ }^{[4]}$ & Left thalamus & Sepsis & Staphylococcus sp. \\
\hline \multirow[t]{2}{*}{ Nakai et al. ${ }^{[5]}$} & Left temporal & Unknown & Gram-positive cocci \\
\hline & Right occipital & Bacteremia & Sterile \\
\hline Siatouni et al. ${ }^{[3]}$ & Left parietal & Urinary tract Infection & Enterococcus faecalis \\
\hline Present case & Right temporal & No focus detected & Escherichia coli \\
\hline
\end{tabular}

no discernible focus of infection. However, there was a delay in recognizing the possibility of an abscess, as the radiological diagnosis of a resolving hematoma was considered. The differential diagnoses of ringenhancing lesions are abscesses (pyogenic, fungal, parasitic), gliomas, lymphomas, metastases, resolving hematomas and radionecrosis. ${ }^{[10]}$ The delay in arriving at a diagnosis and the iatrogenic intraventricular rupture of the abscess that occurred at the time of surgery were the main contributors to the poor outcome in this patient.

\section{Conclusion}

There should be a high index of suspicion for a brain abscess following an intracerebral hemorrhage if the clinical and radiological picture differs from the expected course of a resolving hematoma.

\section{References}

1. Moorthy RK, Rajshekhar V. Management of brain abscess: An overview. Neurosurg Focus 2008;24:3-9.
2. Dashti SR, Baharvahdat H, Sauvageau E, Chang SW, Stiefel MF, Park MS, et al. Brain abscess formation at the site of intracerebral hemorrhage secondary to central nervous system vasculitis. Neurosurg Focus 2008;24:12-6.

3. Siatouni A, Mpouras T, Boviatsis EJ, Gatzonis S, Stefanatou M, Sakas D. Brain abscess following intracerebral haemorrhage. J Clin Neurosei 2007;14:986-9

4. Nowak DA, Rodiek SO, Topka H. Pyogenic brain abscess following haematogenous seeding of a thalamic haemorrhage. Neuroradiology $2003 ; 45: 157-9$

5. Nakai K, Yamamoto T, Yasuda S, Matsumura A. Brain abscess following intracerebral haemorrhage. J Clin Neurosei 2006;13:1047-51.

6. Amayo EO, Kwasa TO, Musau CK, Mugo N, Wambani J. Primary intracerebral haemorrhage complicated by cerebral abscess: Case report. East Afr Med J 2002;79:163-4.

7. Chen ST, Tang LM, Ro LS. Brain abscess as a complication of stroke. Stroke 1995;26:696-8.

8. Biller J, Adams P Jr, Godersky JC, Johnson R. Preeclampsia complicated by cerebral hemorrhage and brain abscess. J Neurol 1985;232:378-80.

9. Inamatsu J, Kagami H, Nakamura Y, Saito R, Niimi M, Ichikizaki K. Brain abscess developing at the site of preceding intracerebral hemorrhage. J Neurol 2002;249:221-3.

10. Cortese I, Nath A. Case 11: A young woman with ring-enhancing brain lesions. Med Gen Med 2006;8:3-6.

Accepted on 13-02-2009

Source of Support: Nil, Conflict of Interest: None declared. 